\title{
Analytical Calculation of Annular Gap Throttling of Damping Valve
}

\author{
Yijie Chen, ${ }^{1, *}$, Xiaodong $\mathrm{Gao}^{1}$, Xiaoling $\mathrm{Han}^{1}$, Ning Zhao $^{1}$, and Yiqiang Wan ${ }^{1}$ \\ ${ }^{1}$ China North Vehicle Research Institute, Beijing, China
}

\begin{abstract}
For the throttling characteristics of the suspension system damping valve, an analytical method for gap throttling based on turbulent flow state was proposed for the first time. The boundary layer theory was used to give the expression of the three-layer velocity distribution of the fluid under turbulent flow state, and the analytical formula of the gap flow of the parallel plate was derived by the integral theorem. Compared with the numerical solution of CFD software, the error was very small, which verified the correctness of the equation. Based on the structural characteristics of the damping valve, the turbulent flow formula of the concentric annular gap was derived, and the influence of different physical parameters on the differential pressure and flow of the gap was analyzed, which lay the foundation for improving the design precision of the damping valve.
\end{abstract}

\section{Introduction}

In the prior art, the gap throttling can be analysed mainly by the following methods: (1) The analytic calculation of the gap flow using the Hydraulic Engineering Handbook is simple and well known, and has been widely used in engineering design. The viscosity of the oil in the damping device is large, the throttle area is relatively small, but due to the significant increase in the over-flow, the oil is mainly in a turbulent state when it is deformed by the damping valve, which leads to a large calculation error. (2) The differential solution of fluid motion N-S second-order nonlinear non-homogeneous partial differential equations is solved, but the calculation process is too complicated, and only the numerical solution in specific cases can be obtained, which is not suitable for engineering applications; (3) Using fluid dynamics software modeling and post-processing analysis, which can get more accurate numerical solution of relevant parameters, however, it is impossible to draw specific expression, and it is difficult to play important role in the design of the damping valve. ${ }^{[1-2]}$

Based on the above situation, this paper uses the theory of fluid mechanics to deduct, verify and systematically study the analytical formula of turbulent flow gap of damping valve in different assembly modes.

\section{Parallel plate slot throttling analytical calculation}

Fig 1 is a schematic illustration of fluid passing through a parallel plate. ${ }^{p_{1}}$ is the inlet end pressure, $p_{2}$ is the outlet end pressure, $\delta$ is the plate spacing, $l$ is the plate length, $V_{\mathrm{f}}$ is the fluid movement speed, and the pressure difference between the two ends of the plate is $\Delta p=p_{1}-p_{2}$, and the plate thickness in the $z$ direction is set to the unit length.

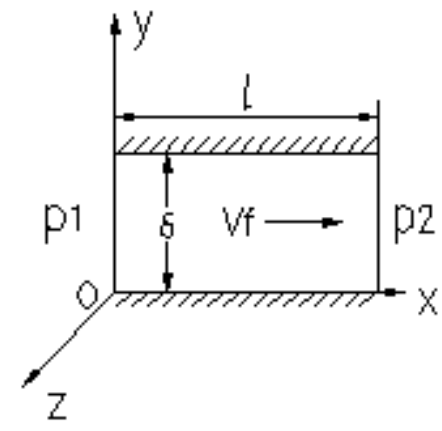

Fig.1. Sketch of parallel-plate.

The formula of the turbulent flow rate of the parallel plate gap is derived by the fluid velocity boundary layer theory.

(1) Velocity distribution law

As the distance of the fluid in the turbulent state changes from the wall surface, the degree of influence of the viscous shear stress and the turbulent additional shear stress on its flow also changes. From the wall surface to the center of the gap, as $y$ increases, the effect of the viscous shear stress gradually decreases, however, the additional shear stress of turbulent flow has an increasing influence on the velocity flow. According to the turbulent flow-average momentum equation, the viscosity vorticity theory and the Prandtl mixing length theory, the sum of the two is a constant and equal to the wall shear stress ${ }^{[3-4]}$ :

\footnotetext{
*Corresponding author: chenyijie1206@163.com
} 


$$
\mu \frac{d \bar{u}}{d y}+\rho l_{\mathrm{p}}^{2}\left(\frac{d \bar{u}}{d y}\right)^{2}=\tau_{\mathrm{w}}
$$

Where $\bar{u}$ is the time-averaged velocity distribution, $\tau_{\mathrm{w}}$ is the wall shear stress, $l_{\mathrm{p}}$ is the Prandtl mixing length, $\rho$ is the fluid density, and $\mu$ is the hydrodynamic viscosity.

The wall turbulence velocity boundary layer is generally divided into an inner layer and an outer layer, and the inner layer includes an adhesive bottom layer, a transition layer and a logarithmic layer; The outer layer includes a trailing layer and a sticky top layer. In a closed gap flow, the influence of the outer layer may not be considered. Fig 2 is the experimental curve of the average velocity distribution in the boundary layer ${ }^{[5]}$ :

$$
\bar{u}^{+}=\frac{\bar{u}}{V^{*}}, \quad y^{+}=\frac{y}{l^{*}}, \quad l^{*}=\frac{v}{V^{*}}, \quad V^{*}=\sqrt{\frac{\tau_{\mathrm{w}}}{\rho}}
$$

Where $\bar{u}^{+}$is a dimensionless velocity, $y^{+}$is a dimensionless to wall distance, $l^{*}$ is the characteristic length, and $V^{*}$ is the wall shear stress velocity.

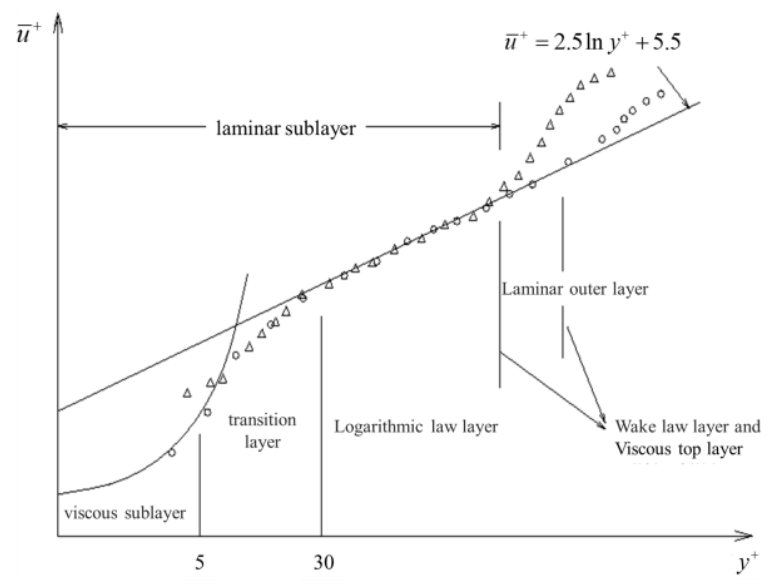

Fig. 2. Boundary layer velocity average distribution curve.

(2) Analytical formula of single-wide parallel plate turbulent flow

Integral solution for single-wide parallel plate turbulent flow analytical equation:

$$
Q_{d}=2\left(\int_{0}^{5} \bar{u}_{c}^{+} d y^{+}+\int_{5}^{30} \bar{u}_{g}^{+} d y^{+}+\int_{30}^{\frac{\delta}{2}} \bar{u}_{d}^{+} d y^{+}\right)
$$

Where $\bar{u}_{c}^{+}$is the viscous bottom layer velocity distribution, $\bar{u}_{g}^{+}$is the transition layer velocity distribution, and $\bar{u}_{d}^{+}$is the logarithmic layer velocity distribution.

The time-average velocity distribution and thickness expression of each layer in the above turbulent state are brought into the integral form and simplified:

$$
Q_{d}=-127.79 v+\left[3+2.5 \ln \left(\frac{\delta}{2 v} \sqrt{\frac{\tau_{w}}{\rho}}\right)\right] \delta \sqrt{\frac{\tau_{w}}{\rho}}
$$

The total friction generated between a single wide parallel plate and a fluid is:

$$
F_{\mathrm{m}}=2 l \tau_{\mathrm{w}}
$$

The pressure difference across the gap is:

$$
F_{y}=\Delta p \delta
$$

According to the principle that the pressure difference is balanced with the frictional force on the plate, the wall shear stress expression can be obtained[6]:

$$
\tau_{w}=\frac{\Delta p \delta}{2 l}
$$

Bring into (3) and organize it:

$$
Q_{\mathrm{d}}=-127.79 v+\left[3+2.5 \ln \left(\sqrt{\frac{\Delta p \delta^{3}}{8 v^{2} l \rho}}\right)\right] \sqrt{\frac{\Delta p \delta^{3}}{2 l \rho}}
$$

The above formula is the flow analysis formula of the parallel plate gap in the turbulent flow state, and based on this, the analytical equation of the concentric annular gap turbulent flow can be derived.

\section{Concentric calculation of concentric annular gap throttling}

When the throttle plate is mounted on the inside of the piston and subjected to a uniform load, the oil flows through the annular gap between the valve plate and the piston wall, and the pressure difference is generated; In the case of reasonable tolerance size design, it can be approximated as concentric annular gap. Fig 3 is schematic view of the fluid passing through concentric annular gap, $r_{\mathrm{n}}$ is the inner radius of the annular gap, and the remaining parameters are defined as above.

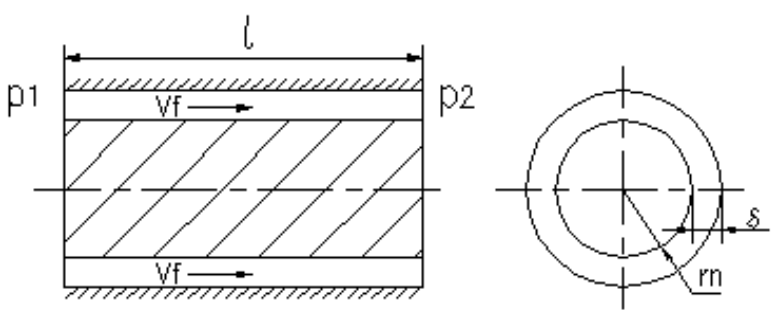

Fig. 3. Concentric annular gap schematic

After the concentric annular gap is unfolded, it is equivalent to a parallel plate slit having the width $B_{\mathrm{k}}=2 \pi r_{\mathrm{n}}$, the circumferential integral of the formula (6) can be used to sort out the turbulent flow of the concentric annular gap:

$Q_{\mathrm{t}}=2 \pi r_{\mathrm{n}}\left\{-127.79 v+\left[3+2.5 \ln \left(\sqrt{\frac{\Delta p \delta^{3}}{8 v^{2} l \rho}}\right)\right] \sqrt{\frac{\Delta p \delta^{3}}{2 l \rho}}\right\}$ 


\section{Analytical verification and analysis of crevice flow}

In the prior art, since there is no known empirical formula for the turbulent flow rate of the gap, so the proposed analytical equation (6) is verified by the fluid dynamics software FLUENT which is widely used in engineering and has high calculation accuracy. First, use the pre-processing module GAMBIT to establish the physical model of the gap, and divide the rectangular grid, import the post-processing solver, given the velocity boundary condition, set the residual to 0.001 and select the k-epsilon turbulence model for numerical calculation.

The relevant parameters for the gap and oil are set as follows: gap width is $\delta=0.004 \mathrm{~m}$, long gap is $l=0.1 \mathrm{~m}$, Oil kinematic viscosity is $v=1 \times 10^{-5} \mathrm{~m}^{2} / \mathrm{s}$, density is $\rho=890 \mathrm{~kg} / \mathrm{m}^{3}$. The flow velocity when taking the oil through the gap is $V_{\mathrm{f}}=15 \mathrm{~m} / \mathrm{s}, 20 \mathrm{~m} / \mathrm{s}, 25 \mathrm{~m} / \mathrm{s}, 30 \mathrm{~m} / \mathrm{s}$, Ensure Reynolds number $\operatorname{Re}>1100$, The pressure difference comparison results are shown in Tab1.

Tab1. Parallel plate gap pressure difference comparison.

\begin{tabular}{|c|c|c|c|c|}
\hline$V_{\mathrm{f}} /(\mathrm{m} / \mathrm{s})$ & $\Delta p_{\mathrm{c}} / \mathrm{Mpa}$ & $\Delta p_{\mathrm{w}} / \mathrm{Mpa}$ & $\Delta p_{\mathrm{F}} / \mathrm{Mpa}$ & $\gamma / \%$ \\
\hline 15 & 0.010 & 0.040 & 0.042 & 5.0 \\
\hline 20 & 0.013 & 0.066 & 0.070 & 5.7 \\
\hline 25 & 0.017 & 0.096 & 0.100 & 4.0 \\
\hline 30 & 0.020 & 0.130 & 0.140 & 7.1 \\
\hline
\end{tabular}

In the table, is the turbulent flow formula to calculate the differential pressure, is the FLUENT software to calculate the differential pressure, is the relative deviation between the turbulent flow formula and the FLUENT software, and the laminar flow formula calculates the differential pressure as:

$$
\Delta p_{\mathrm{c}}=\frac{12 \mu l Q_{\mathrm{d}}}{\delta^{3}}
$$

It can be seen from the data in the table that the calculation results of the parallel plate gap turbulent flow formula and the numerical solution of the fluid dynamics software are relatively small, thus verifying the correctness of the above derivation process and the formula. However, the analytical solution calculated by the formula of the laminar laminar flow rate has a large error and the numerical value is obviously small, it is shown that the formula (10) is not suitable for the case of gap throttling under turbulent flow conditions, and it also shows that the self-derived turbulent flow formula has application background and practical value.

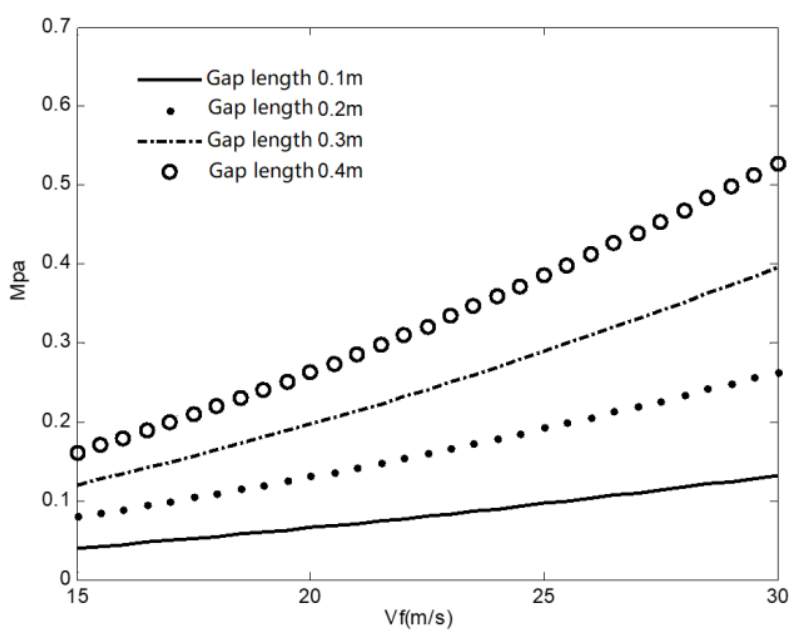

Fig.4. Pressure difference between different gap lengths.

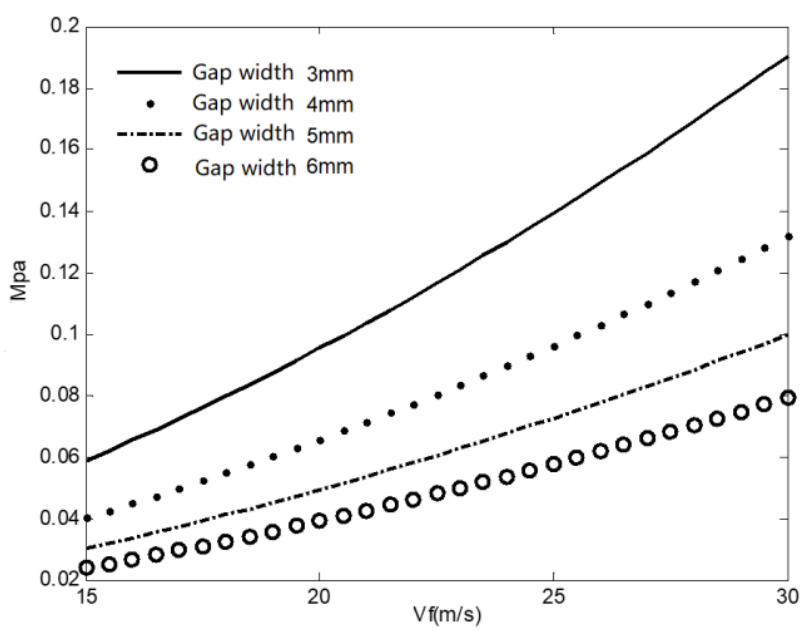

Fig.5. Pressure difference between different gap widths.

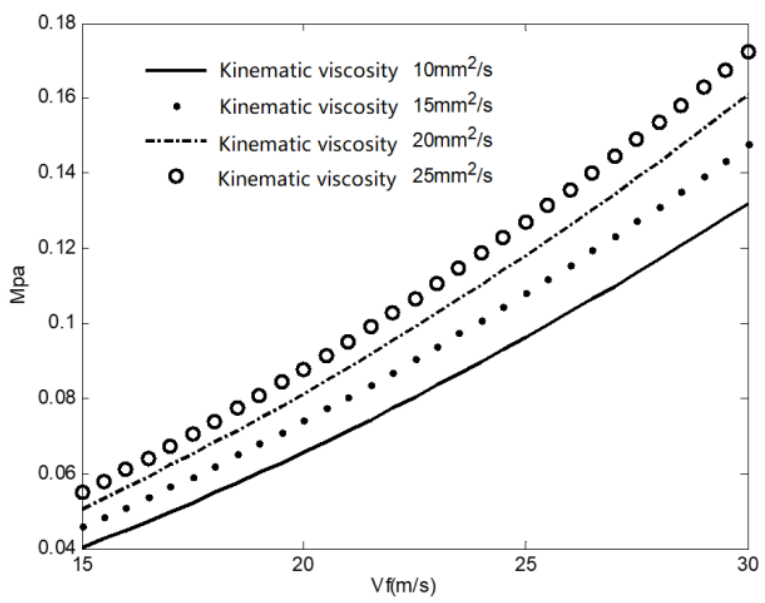

Fig.6. Pressure difference between different kinematic viscosities. 


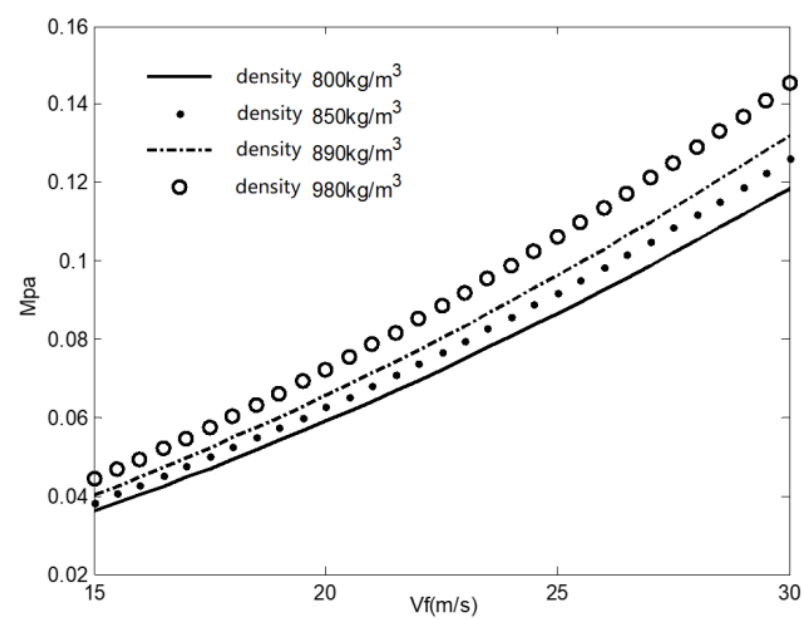

Fig.7. Pressure difference between different oil densities.

The turbulent flow formulas (6) and (10) are used to analyze the influence of the gap structure parameters and the oil-related characteristics on the differential pressure when the oil-related characteristics change within a certain range. Set the oil flow rate to $V_{\mathrm{f}}=15 \mathrm{~m} / \mathrm{s}: 30 \mathrm{~m} / \mathrm{s}$, The calculation results are shown in Figure 4-7,it can be seen that as the length of the gap increases, the contact area between the oil and the wall becomes larger, and the total friction generated also increases; As the viscosity of the oil moves, the attraction between the molecules of the oil and the friction with the wall gradually increase; As the density of the oil increases, the probability of collision during molecular motion increases, and more energy can be consumed. Due to the above factors, the pressure difference of the oil at the same overcurrent speed shows an upward trend. In addition, as the gap width increases, the throttling effect gradually decreases, and the pressure difference of the oil at the same moving speed is significantly reduced.

\section{Conclusions}

For the damper valve form of the slit throttling, the analytic formula of the turbulent flow under the annular gap structure is deduced and systematically studied by the theory of fluid mechanics. The work carried out is as follows:
Using the fluid dynamics boundary layer theory, the closed gap flow in the turbulent flow state is divided into three layers of the adhesive bottom layer, the transition layer and the logarithmic layer in order from the wall to the far side. The expression of the time-averaged velocity distribution of each fluid in each layer of the fully developed section is given, and the analytic formula of single-wide parallel plate turbulent flow is derived by integral. Compared with the numerical solution of the pressure difference of FLUENT at different speeds, the correctness of the formula is verified. After that, the influence of relevant parameters on the pressure difference at both ends of the gap is further analyzed, which provides reference for the design of the damping valve. Based on the formula of singlewidth parallel plate turbulent flow, the analytical formula of layer turbulent flow in concentric annular gap is derived.

\section{References}

1. Zuo L, Zhang PS. Energy Harvesting, Ride Comfort, and Road Handling of Regenerative Vehicle Suspensions[J]. Journal of Vibration \& Acoustics, 2013, 135 (1): 48-65.

2. Wang $\mathrm{R}$, Chen $\mathrm{Z}, \mathrm{Xu} \mathrm{H}$, et al. Modelling and validation of a regenerative shock absorber system[C]. International Conference on Automation and Computing, 2014: 32-37.

3. Maravandi A, Moallem M. Regenerative Shock Absorber Using a Two-Leg Motion Conversion Mechanism[J]. IEEE/ASME Transactions on Mechatronics, 2015, 20 (6):2853-2861.

4. Roshan YM, Maravandi A, Moallem M. Power Electronics Control of an Energy Regenerative Mechatronic Damper[J]. IEEE Transactions on Industrial Electronics,2015, 62 (5): 3052-3060.

5. Sabzehgar R, A Maravandi, M Moallem. Energy Regenerative Suspension Using an Algebraic Screw Linkage Mechanism[J]. IEEE/ASME Transactions on Mechatronics, 2014, 19 (4): 1251-1259. 